\title{
Supervisión de casos: Depresión persistente
}

\author{
Robin Shapiro \\ Seattle, Washington \\ Arne Hofmann \\ Instituto EMDR, Alemania \\ Earl Grey \\ Universidad de Walden, Pittsburgh, Pennsylvania
}

\begin{abstract}
Supervisión de casos es un nuevo apartado regular del Journal of EMDR Practice and Research. En este artículo, una terapeuta de desensibilización y reprocesamiento por movimientos oculares (EMDR) describe brevemente un caso complicado en el cual un hombre, "George", fue derivado a tratamiento EMDR por una depresión que había comenzado hacía más de dos años. Después de haber procesado completamente con EMDR todos los recuerdos traumáticos que había descrito, George continúa gravemente deprimido y la terapeuta pregunta cómo puede proceder de manera eficaz con el tratamiento. Tres especialistas responden por escrito. La primera especialista, Robin Shapiro, describe una lista exhaustiva de posibles etiologías, que incluyen causas de apego, de trauma temprano, genéticas y otras causas biológicas, además del tratamiento de EMDR, de estados del ego o médico adecuado para cada una. El segundo experto, Arne Hofmann, analiza el tratamiento impartido y hace sugerencias de blancos alternativos para el tratamiento y sugiere que la terapeuta podría trabajar con la creencia "nada cambiará" y probar con el protocolo invertido de EMDR. El tercer experto, Earl Grey, recomienda que el terapeuta se centre en trabajar los traumas de "t" pequeña, aún si el paciente indica que apenas tiene perturbación y explica la manera de desarrollar e implementar una "secuencia reparadora de blancos de la vida".
\end{abstract}

Palabras clave: EMDR; depresión; supervisión; tratamiento

\section{Pregunta del terapeuta}

Me derivaron a "George" para tratamiento de desensibilización y reprocesamiento por movimientos oculares (EMDR) por una depresión severa que había comenzado hacía más de dos años. He hecho unas 10 sesiones de EMDR (F. Shapiro, 2001) con George. Los blancos han incluido hechos perturbadores que ocurrieron durante su hospitalización involuntaria por un trastorno del estado de ánimo y otros acontecimientos que precedieron a su hospitalización. Ya no refiere angustia relacionada con las hospitalizaciones ni los eventos previos, ya no tiene recuerdos intrusivos sobre ellos y ya no se preocupa obsesivamente sobre ellos. Su cognición negativa original $(\mathrm{CN})$ era "soy una mala persona" y esto ha cambiado a "estoy bien" con una validez alta. Ya no tiene síntomas traumáticos porque todos parecen haberse resuelto completamente con EMDR.

Sin embargo, esta resolución no le produce ninguna satisfacción a George porque sigue estando gravemente deprimido.

Ha sido incapaz de volver a trabajar y ha mantenido su estado de discapacitado. Indica que no siente placer alguno en su actividad diaria y expresa desesperanza sin ninguna expectativa de mejoría en el futuro. Le cuesta mucho movilizarse para hacer cualquier cosa, aunque da paseos diarios con su mujer, que es un apoyo para él. Está tomando medicación: antidepresivos, antipsicóticos y estabilizadores del estado de ánimo.

This article originally appeared as Shapiro, R., Hoffman, A., \& Grey, E. (2013). Case consultation: Unremitting depression. Journal of EMDR Practice and Research, 7(1), 39-44. Translated by Miriam Ramos Morrison. 
Después de completar nuestro trabajo con sus recuerdos traumáticos, reenfocamos nuestra atención a su depresión, convirtiéndola en blanco con una nueva CN de "soy un fracaso". Hicimos ejercicios de flotar hacia atrás con su emoción y su CN actual (soy un fracaso), pero no surgieron recuerdos y él insiste en que su vida estaba bien antes de los eventos que precedieron a su hospitalización, la cual hemos procesado y ya no le molesta. George habla de una infancia ordinaria sin complicaciones y sin incidentes angustiosos ni experiencias previas en las que se haya sentido como un fracaso. Insiste en que no hay ningún evento vital que le cause perturbación emocional alguna y que ya no se preocupa obsesivamente sobre el pasado; ahora simplemente lo hace sobre lo deprimido que está y su creencia de que nada va a cambiar.

Hemos hecho EMDR sobre su experiencia actual de depresión, con un par de blancos (la imagen de sí mismo sintiéndose desesperado y las pérdidas asociadas) pero de hecho parece que esto le deprime más porque sus asociaciones tienden a ser muy negativas. No podemos trabajar con ningún tipo de recursos porque George es incapaz de imaginar nada positivo. Nada parece cambiar con el procesamiento y se mantiene inmerso en su profunda depresión.

Parece que nuestro fracaso con EMDR refleja su sensación de fracaso personal, con lo cual tengo dudas sobre si debería continuar. ¿Hay algo que podamos hacer?

\section{Respuesta de la especialista $n^{\circ} 1$, Robin Shapiro}

Según van der Kolk et al. (2007), EMDR es un tratamiento eficaz y rápido para el trastorno de estrés postraumático (TEPT) y sus síntomas depresivos resultantes. Con el trauma ocurrido en la edad adulta, funciona muy rápidamente. Los traumas ocurridos en la infancia a menudo tardan más en despejarse. Sin embargo, la depresión puede tener otras causas y el tratamiento más adecuado dependerá de su etiología.

La depresión se está conceptualizando cada vez más como un proceso corporal inflamatorio. La enfermedad fisica o el trauma y el trauma emocional o el duelo pueden comenzar el proceso, dicen Hedaya (2009); Krishnadas y Cavanagh (2012); Raison et al. (2012); Wager-Smith y Markou (2011); Weil (2011); Zunsain, Hepgul y Pariante (2012); y muchos otros. Encuentran que la gente con tendencia a sufrir ansiedad tienen más probabilidades de estresarse y luego deprimirse. ¿Recuerdas la última vez que tuviste el típico proceso inflamatorio de fiebre alta? Tu cuerpo entró en modo dorsal vagal (colapso) para obligarte a que te tumbases y sanases. Si no tuvieras la excusa de la fiebre y estuvieras exhausto, sin apetito, no pudieras sentir placer y fueras incapaz de conectar bien con otras personas, puede que te diagnosticáramos con depresión. Según aquellos que proponen la conexión entre depresión e inflamación, el cuerpo de una persona con depresión entra en un estado similar en un intento de autocuración.

Con lo cual, ¿qué hacemos con George? Dado que le hospitalizaron por un trastorno del estado de ánimo, queremos saber su historial completo genético, de apego y de trauma. Necesitas averiguar cualquier cambio o factores de estrés que haya en su vida actual. Para obtener esta información, podrías hacer un genograma para descubrir si alguien más en la familia tiene historia de depresión, ansiedad u otro trastorno del estado de ánimo; lo que George ha experimentado personalmente con su trastorno; alguna interrupción del apego al principio de su vida; si habido periodos de estrés crónico o trauma en la vida de George; o si ha habido pérdidas grandes y no resueltas en su vida. Si encuentras los problemas de apego, pérdida, estrés o trauma, los procedimientos de EMDR pueden ser suficientes para enfrentarse al problema. Si se trata de un problema de apego, es necesario asumir que la terapia será más larga y posiblemente habrá que focalizarse en escenas de la madre y el bebé, luego de la madre y el niño y luego de la madre y el niño más mayor mediante el uso del modelo de desarrollo estratégico de EMDR de Kitchur (2005): "Imagina que eres un bebé en brazos de su madre. Mírala a la cara. ¿Qué emoción notas ahora? ¿Qué sensación?” Y usar el protocolo estándar para disipar lo que se encuentre. Es posible ofrecer recursos mediante el trabajo de "construcción de un yo seguro" de Steele (2007): "Imagínate a ti mismo como adulto, sosteniendo en brazos a tu yo bebé”. O el trabajo de Knipe (2009): "Haz que tu adulto mire a ese niño con ojos de amor". Haces frente a los problemas y ves como poco a poco la persona va saliendo de la niebla.

Si es trauma crónico o trauma de hace mucho tiempo, aún será una terapia más larga de 10 sesiones. Comienza con los traumas iniciales y trabaja hacia el presente. Cuanto más vayas disipando, más debería ir desapareciendo la depresión.

Si existe estrés crónico, usa las tres vertientes para disipar los factores de estrés del pasado y el presente y después utiliza plantillas de futuro para programar nuevas respuestas ante estos factores de estrés.

Si es un trastorno bipolar, vas a educar a tu paciente sobre la enfermedad, encontrar el mejor psiquiatra de la ciudad y trabajar con tu paciente para que tome medicación, suplementos vitamínicos (omega-3s, aspirina y otros anti-inflamatorios pueden ser útiles), tenga un horario regular y aprenda a gestionar su 
enfermedad crónica. La tercera vertiente de EMDR, la plantilla de futuro, puede ser útil para practicar un buen autocuidado internamente. La primera y la segunda vertiente de EMDR pueden ayudar a despejar la angustia sobre el diagnóstico. Las personas ansiosas tienen más posibilidades de estresarse y deprimirse.

Si no ves ninguna evidencia de trauma actual o pasado, problemas de apego ni trastorno bipolar, es hora de centrarse en otras etiologías físicas. ¿Has estado George tomando drogas estimulantes como cocaína o anfetaminas? Recuerda que todo lo que sube puede estrellarse contra el suelo, haciendo que la gente se sorprenda de encontrarse en estados muy bajos (dorsal vagal). El éxtasis y las anfetaminas agotan la serotonina del cerebro y generan una incapacidad para encontrarse bien. Estas son las causas obvias. En relación a causas físicas menos evidentes, necesitas enviar a tu paciente a un buen médico que busque las causas subyacentes de esta depresión incurable tan duradera. A muchos psiquiatras se les escapan estos problemas físicos, con lo cual derivarle a un buen internista puede hacer milagros. Esto es lo que debería buscar el médico:

- Temas hormonales: hipotiroidismo, testosterona baja o insuficiencia de otras hormonas

- Pequeñas infecciones subyacentes

- Desequilibrios nutricionales, especialmente deficiencia de vitamina $\mathrm{D}$

- Tensión sanguínea excesivamente baja

- Lesión crónica o dolor que puede o bien crear una respuesta inflamatoria en todo el cuerpo que genere una depresión o una reacción opiácea endógena que imita/genera depresión

- Cualquier señal de tumores en el cerebro, glándulas o cualquier lugar que pueda causar una respuesta depresiva

- Cualquier enfermedad sistémica inflamatoria no enumerada previamente

Si George está deprimido por alguna de estas razones, puedes usar EMDR para disipar la angustia de estar enfermo y apoyarle para que haga lo que necesite hacer para sanar y mejorar su estado de ánimo. Céntrate en su angustia sobre el diagnóstico (si lo hay) o instala el alivio de que haya una causa conocida que tiene arreglo. Utiliza plantillas de futuro para apoyarle en hacer lo que necesita hacer. Independientemente de la causa de su depresión, no te rindas con George. La conexión social mejora el estado de ánimo. El abandono le dice que tiene razón al sentirse desesperado. Déjale saber que estás de su lado. Déjale saber que sabes lo angustioso que es estar tan deprimido. Mantente a su lado mientras descubrís qué ocurrió y qué podéis hacer con ello.

\section{Respuesta del especialista $n^{\circ} \mathbf{2}$, Arne Hofmann}

El tratamiento de una depresión persistente es un reto importante y EMDR no es una solución mágica para todos los casos. Sin embargo, EMDR puede ser una herramienta muy valiosa en el tratamiento de aquellos pacientes que o bien se mantienen sin mejoría o están siendo tratados con terapia electroconvulsiva. George parece sufrir de una depresión muy grave que no remite con una medicación compleja y una psicoterapia bien planificada. Si yo hiciese supervisión con el terapeuta, intentaría desarrollar los siguientes pasos posibles siguiendo estas líneas:

1. Dado que todo el tratamiento actual no le ha aportado un alivio significativo a George, echaría otro vistazo a los contribuyentes somáticos. Hay varias medicinas y sustancias que pueden causar o sostener una depresión y que deberían sustituirse o dejar de tomarse. Los fármacos contra la hipertensión (especialmente los agentes betabloqueantes) no sólo reducen la presión sanguínea, sino que a veces tienen un efecto depresogénico en sí mismos. Además, el alcohol y las benzodiazepinas, especialmente en una situación de adicción latente u obvia, se encuentran entre los culpables más frecuentes de fomentar o incluso iniciar un episodio depresivo. Como tercer posible contribuyente somático, debería descartarse el camaleón de la medicina interna, los problemas de tiroides, especialmente el hipotiroidismo. Los problemas de tiroides pueden imitar diversas enfermedades internas, psicosomáticas y psiquiátricas, y la depresión es una de ellas. Esto ocurre especialmente en el caso de que el estabilizador del estado de ánimo que George está tomando sea litio. Se sabe que el litio afecta a la función de la tiroides y controla la hormona estimulante de la tiroides (TSH); normalmente se recomienda comprobar el funcionamiento del ciclo de retroalimentación de la tiroides.

2. Mientras se trabaja con los acontecimientos del pasado en su depresións, la estancia involuntaria ("traumática”) en el hospital es solamente uno de los eventos en los que George necesita trabajar. Su depresión (¿y tendencia suicida?) ha comenzado antes y, normalmente, existen acontecimientos relacionados muy de cerca con el comienzo de un episodio depresivo. Muchos de estos eventos disparadores de episodios están relacionados con pérdidas y humillaciones y a menudo podrían clasificarse como "trauma de apego". Además, si George ha sufrido episodios depresivos previos, los eventos que 
inician estos episodios previos y los posibles eventos traumáticos asociados con estos episodios deberían procesarse también (incluso si la puntuación en la escala de unidades subjetivas de perturbación [SUD] es solo de 3 o 4 y el recuerdo ya no es intrusivo). Asumo que el terapeuta ya ha hecho esto y tan sólo lo añado para completar mi respuesta.

3. Una de las mejores maneras de trabajar con algunos clientes con depresión es el trabajo con los sistemas de creencias. El terapeuta ha intentado hacer esto, pero el floatback/puente afectivo, así como la pregunta de los eventos asociados con la experiencia de "soy un fracaso" ("recuerdos de prueba" según de Jongh) no resultaron en la aparición de nuevos eventos estresantes. Sin embargo, si se puede alterar el estado depresivo (por ejemplo, con técnicas de activación de recursos), yo intentaría echarle otro vistazo al sistema de creencias más adelante. En ocasiones, el estado actual de un paciente con depresión no permite hacer más trabajo con recuerdos. Más adelante en el proceso de terapia, sin embargo, se puede acceder a y trabajar con los mismos sistemas de creencias y recuerdos mucho más fácilmente.

4. Hay algunos casos de depresión (la mayoría de ellos casos crónicos) en los que, al igual que en casos complejos de TEPT, el trabajo con recuerdos pasados no sirve para ayudar al paciente a salir de su "estado" actual altamente sintomático. En algunos de estos casos, o bien el pasado es tan complejo (y lleno de amnesia) que no puede procesarse al principio, o bien el presente es aún tan estresante que cada vez que nos centramos en el pasado, el presente negativo se cuela en el proceso terapéutico. Para estos casos, hemos desarrollado un protocolo invertido de EMDR (Hofmann, 2009). En vez de enfocarnos primero en el pasado, después en el presente y luego en el futuro (como hacemos en el protocolo estándar de EMDR), nos centramos primero en los posibles problemas de los próximos días (futuro) y en el presente antes de trabajar más tarde con el pasado. Para hacer esto con éxito, es necesario activar la red de recursos. Los recursos imaginarios (como el lugar seguro) son tan sólo una de las diversas posibilidades de desarrollo de recursos que aquí solamente puedo mencionar (Korn y Leeds [2002] podrían explicar esto mucho mejor). En mi opinión, un recurso es una red activada (de memoria) que se enlaza con una emoción positiva que el paciente puede sentir en su cuerpo. No es necesario utilizar la imaginación para este tipo de trabajo de recursos. Los pequeños cambios en las sensaciones corporales pueden surgir durante una sesión de terapia y marcar una gran diferencia para el paciente. Sin embargo, este tipo de trabajo con recursos puede implicar más tiempo que las 10 sesiones que George ya ha tenido. Algunos pacientes necesitan muchos meses para salir de un estado en el que los recursos han estado completamente agotados. La mayoría de los recursos que desarrollan giran en torno a personas de apego (lo que incluye probablemente al terapeuta), animales de apego, pequeños éxitos durante la terapia y cosas así. Tal vez en alguna de estas áreas, el terapeuta y yo podríamos encontrar algo que ayudase a dar el próximo paso en el tratamiento de George.

\section{Respuesta del especialista $n^{\circ}$ 3, Earl Grey}

Basándonos en la información que se ha aportado, los siguientes pasos podrían añadir un aumento del resultado positivo del tratamiento. Recomiendo que el terapeuta conceptualice muy claramente los problemas actuales mediante el uso de los postulados del modelo de procesamiento adaptativo de información (PAI) de F. Shapiro (2001), seguido del desarrollo y reprocesamiento de los eventos de la vida del paciente mediante el uso de un plan reparador de secuencia de blancos de la vida (Grey y Morrow, 2011).

\section{Conceptualización del caso desde el procesamiento adaptativo de la información}

George ha buscado tratamiento EMDR por una reacción traumática ante una hospitalización involuntaria. Él define su hospitalización como un trauma de " $T$ " grande. Este evento perturbador ha exacerbado su depresión, que parece haber sido persistente durante la edad adulta. George indica que no hay otros traumas de "T" grande a lo largo de su vida. Debido a la naturaleza del ser humano, las personas tienen experiencias con estrés y perturbación subclínicos (Stewart-Grey, 2008). Es más que probable que las perturbaciones subclínicas o "traumas de t pequeña" estén alimentando en parte los síntomas depresivos de George. Dado que los eventos alimentadores probablemente no son traumáticos, George no los está identificando como factores contribuyentes. La longevidad de los síntomas depresivos de George también se fomenta por el hecho de que su $\mathrm{CN}$ se encuentra dentro de la temática del valor personal, descrito por F. Shapiro (2001) como defecto de responsabilidad (Grey, 2011; Stewart-Grey, 2008). Es más, parece haber factores químicos genéticos puestos en evidencia por la necesidad de tomar medicación psicotrópica. Abordar la intervención química está fuera del ámbito de esta respuesta. 
Finalmente, George está indicando que su pasado no tiene eventos de importancia, su presente está lleno de fracaso y su futuro no tiene esperanza. Con la generalización de estos traumas de t pequeña, las posibles influencias genéticas y el desafio de que no haya recuerdos de traumas de T grande a través de las tres vertientes, George no sería un candidato para un protocolo tradicional de EMDR. Para tratar a George de manera eficaz, se recomienda que el terapeuta incluya un protocolo reparador sintetizado dentro de las ocho fases tradicionales del tratamiento EMDR. Hay pruebas de que George tiene deseos de encontrarse bien, como muestran su apego hacia su mujer y el hecho de que participa en estimulación bilateral (paseos diarios). La estimulación bilateral, en general, activa el lóbulo frontal (Hammond, 2008; Squire et al., 2008). La disposición de George de participar en su relación y en sus paseos, aporta las bases para apoyar la pertinencia de un enfoque de tratamiento reparador de EMDR.

\section{Plan de secuenciación del desarrollo}

Hablar con George sobre las etapas del desarrollo vital ayudará a identificar pequeños traumas $t$ que aparentemente son inaccesibles. El objetivo de este proceso es identificar las tareas del desarrollo que probablemente hayan necesitado de ayuda externa de un cuidador, porque estos son puntos en la vida de la persona donde puede que no haya sido capaz de desarrollar un locus de control interno y un apego significativo hacia sí mismo. El resultado normal de los pequeños traumas $t$ que resultan de las tareas del desarrollo es un locus interno no adaptativo del yo con un locus de control externo extremo que se refleja en depresión y creencias negativas destructivas sobre el yo (Davies, 2004; Lewis \& Elman, 2008).

En EMDR, un entretejido cognitivo que se utiliza con frecuencia aborda este problema, al hacer que el paciente procese una interacción imaginaria entre yo niño y el yo adulto. F. Shapiro (2001) indica que un entretejido cognitivo en el que se usa al yo niño y al yo adulto puede ayudar al paciente a "reconocer que ya no es un niño vulnerable” (p. 257).

Este procedimiento es un elemento central en el desarrollo de un plan de secuenciación de blancos en el que cada blanco se procesa con un enfoque en reparar la relación entre las dos perspectivas de uno mismo. La perspectiva dicotómica más frecuente es un yo grande y un yo pequeño. Cuando lidiamos con una persona con hijos, también puede ser eficaz trabajar con el yo padre o madre frente al yo individual. El objetivo es tener dos elementos del yo: uno que posea las habilidades necesarias para apoyar, nutrir y proteger y el otro que esté abierto aceptar a el apoyo, la nutrición y la protección. Este proceso se basa en el desarrollo de la personalidad del niño y es un elemento clave en el desarrollo de nuestras percepciones como adultos (Davies, 2004; Grey, 2010; Grey y Morrow, 2011; Nico y Daprati, 2009).

Cuando elegimos los eventos diana, el paciente y el terapeuta solamente van a seleccionar experiencias que fomenten un gran locus de control interno con el uso de ambos conceptos del yo (esto es, grande y pequeño). Algunos eventos normales del desarrollo que dan lugar a que el niño dependa de un locus de control externo son: (a) "rayo de luz", la primera mirada entre la madre y el bebé directamente después del parto; (b) aprender a estar de pie de forma independiente; (c) aprender a hablar; (d) aprender a caminar; (e) tener un elemento del yo que proteja al otro, lo que aumenta la seguridad interna; (f) actividades de juego exitosas; y (g) eventos vitales específicos que el paciente identifica como centrales en su vida. Estas son solamente sugerencias basadas en el desarrollo humano típico. El paciente puede identificar estos eventos vitales como neutrales o positivos. Los eventos vitales del desarrollo han de negociarse entre terapeuta y paciente. La lista que he ofrecido es una lista de sugerencias de tareas vitales del desarrollo humano. Una vez que hayamos identificado los eventos de la secuenciación de blancos del desarrollo, usaremos el protocolo tradicional de EMDR. El terapeuta va a querer comprobar cada uno de los blancos mediante los procedimientos de protocolo estándar para montar el blanco (F. Shapiro, 2001). Si existen una CN y cierta perturbación, entonces el terapeuta querrá estar preparado para usar el entretejido que se ha sugerido previamente dentro del protocolo de reprocesamiento de todo el pasado.

En el caso de George, también podríamos usar EMDR para procesar sus paseos diarios con su esposa como recurso. Un punto útil para comenzar puede ser usar sus paseos diarios y añadir una parte adicional del yo (George pequeño y George grande) para ayudar a construir el apego relacional con el yo interno de George. La relación de apoyo previamente establecida, además de la naturaleza primitiva del caminar y el desarrollo del caminar, puede ayudar a crecer hacia una resolución reparadora. La intención de este plan de secuenciación del desarrollo es aumentar el apego adaptativo hacia el yo interno de uno mismo. Este proceso se recomienda cuando un paciente presenta deficiencias aparentes en el locus de control interno y no tiene eventos identificados traumáticos o angustiosos sobre los que se pueda hacer blanco. 


\section{Referencias}

Davies, D. (2004). Child development: A practitioner's guide (2nd ed.). New York, NY: Guilford Press.

Grey, E. (2010). Use your brain: A neurologically driven application of REBT with children Journal of Creativity in Mental Health, 5(1), 54-64.

Grey, E. (2011). A pilot study of concentrated EMDR: A brief report. Journal of EMDR Practice and Research, 5(1), 14-24.

Grey, E., \& Morrow, R. (2011, September). Human neuroecology: Attachment, survival, and reflexes in couples. Paper presented at the annual meeting of the American Association of Marriage and Family Therapists, Fort Worth, TX.

Hammond, C. (2008). Cellular and molecular neurophysiology (3rd ed.). Burlington, MA: Academic Press.

Hedaya, R. J. (2009, March). Depression, inflammation, immunity and infection. PsychologyToday. Retrieved from http: / / www.psychologytoday.com/blog/healthmatters / 200903 / depression-inflammation-immunityand-infection

Hofmann, A. (2009). The inverted EMDR standard protocol for unstable complex posttraumatic stress disorder. In M. Luber (Ed.), EMDR scripted protocols. Special populations (pp. 313-328). New York, NY: Springer.

Kitchur, M. (2005). The strategic developmental model for EMDR. In R. Shapiro (Ed.), EMDR solutions: Pathways to healing (pp. 8-56). New York, NY: Norton.

Knipe, J. (2009). "Shame is my safe place": Adaptive information processing methods of resolving chronic shamebased depression. In R. Shapiro (Ed.), EMDR solutions II: Depression, eating disorders, performance, and more (pp. 49-89). New York, NY: Norton.

Korn, D., \& Leeds, A. (2002). Preliminary evidence of efficacy for EMDR resource development and installation in the stabilization phase of treatment of complex posttraumatic stress disorder. Journal of Clinical Psychology, 58(12), 1465-1487.

Krishnadas, R., \& Cavanagh, J. (2012). Depression: An inflammatory illness? Journal of Neurology, Neurosurgery \& Psychiatry, 83, 495-502. http://dx.doi.org/10.1136/ jnnp-2011-301779

Lewis, J., \& Elman, J. (2008). Growth-related neural reorganization and the autism phenotype: A test of the hypothesis that altered brain growth leads to altered connectivity. Developmental Science, 11(1), 135-155.

Nico, D., \& Daprati, E. (2009). The egocentric reference for visual exploration and orientation. Brain \& Cognition, 69, 227-235.

Raison, C. L., Rutherford, R. E., Woolwine, B. J., Suo C., Schettler P., Drake D.F., . . . Miller A. H. (2012). A randomized controlled trial of the tumor necrosis factor depression: The role of baseline inflammatory biomarkers. Archives of General Psychiatry. Advance online publication. http://dx.doi.org/10.1001/2013 .jamapsychiatry.4. Retrieved from http://www.ncbi .nlm.nih.gov/pubmed/22945416
Shapiro, F. (2001). Eye movement desensitization and reprocessing: Basic principles, protocols, and procedures (2nd ed.). New York, NY: Guilford Press.

Squire, L. R., Berg, D., Bloom, F. E., du Lac, S., Ghosh, A., \& Spitzer, N. C. (2008). Fundamental neuroscience (3rd ed.). Burlington, MA: Academic Press.

Steele, A. (2007). Developing a secure self: An attachment-based approach to adult psychotherapy (2nd ed.). Retrieved from http: / / www.april-steele.ca/

Stewart-Grey, E. (2008). De-stress: A qualitative investigation of EMDR treatment. ProQuest Dissertations \& Theses: Full Text. (UMI No. 3329984).

van der Kolk, B. A., Spinazzola, J., Blaustein, M. E., Hopper, J. W., Hopper, E. K., Korn, D. L., \& Simpson, W. B. (2007). A randomized clinical trial of eye movement desensitization and reprocessing (EMDR), fluoxetine, and pill placebo in the treatment of posttraumatic stress disorder: Treatment effects and long-term maintenance. Journal of Clinical Psychiatry, 68(1), 37-46.

Wager-Smith, K., \& Markou, A. (2011). Depression: A repair response to stress-induced neuronal microdamage that grade into a chronic neuroinflammatory condition? Neuroscience \& Biobehavioral Reviews, 35(3), 742-764.

Weil, A. (2011). The depression-inflammation connection. Retrieved from http://www.huffingtonpost.com/andrew weil-md/depression-and-inflammation_b_1071714.html

Zunszain, P. A., Hepgul, N., \& Pariante, C. M. (2012) Inflammation and depression. Current Topics in Behavioral Neuroscience. Advance online publication.

\section{Conoce a los especialistas}

Robin Shapiro, trabajadora social clínica independiente licenciada, es clínico y consultora aprobada por la asociación internacional de EMDR (EMDRIA) en Seattle, Washington. Ha editado y contribuido a EMDR Solutions: Pathways to Healing (2005) y EMDR Solutions II: Depression, Eating Disorders, Performance and More (2009) y ha escrito Trauma Treatments Handbook: Protocols Across the Spectrum (2010). Es antiguo miembro de la junta directiva del programa de ayuda humanitaria de EMDR (HAP).

El Dr. Arne Hofmann, MD, PhD, es especialista en medicina interna y psicosomática, director del Instituto alemán de EMDR e instructor de EMDR aprobado por EMDR Europa. Ha escrito y editado tres libros de EMDR sobre el diagnóstico y tratamiento de los trastornos disociativos. Es miembro de la comisión nacional alemana sobre las pautas para el TEPT y cofundador de la sociedad de habla alemana para el estudio de estrés traumático (DeGPT). Enseña e investiga a nivel internacional y actualmente es investigador principal de un ensayo controlado aleatorizado multicentro europeo que explora el efecto del EMDR en la depresión crónica y recurrente. 
El Dr. Earl Grey, PhD, es terapeuta profesional licenciado, consultor aprobado por EMDRIA y facilitador de EMDR para el Instituto / HAP en Pittsburgh, Pennsylvania. Es catedrático CORE en la Universidad de Walden, Escuela de ciencias sociales y conductuales, departamento de asesoramiento. Es autor del libro Unify Your Mind sobre la neurociencia del procesamiento de la información. Ha dirigido y publicado investigación de estudios de casos sobre el uso de EMDR con depresión.

La correspondencia sobre este artículo debería dirigirse a Robin Shapiro, 6869 Woodlawn Avenue NE, 204 A, Seattle, WA 98115. E-mail: mdrsolutions@gmail.com; Dr. Arne Hofmann, EMDR Institute Germany, Dolmanstrasse 86b 51427 Bergisch Gladbach, Germany. E-mail: arne-Hofmann@t-online.de 